Itinéraires Itinéraires

Littérature, textes, cultures

2016-2 | 2017

Livre, sérialité et transmédialité

\title{
Le jeu de rôle, une autre forme de narration sérielle?
}

Pen and Paper Roleplaying Game, Another Serial Narration?

Isabelle Périer

\section{OpenEdition}

1 Journals

Édition électronique

URL : http://journals.openedition.org/itineraires/3453

DOI : $10.4000 /$ itineraires.3453

ISSN : 2427-920X

Éditeur

Pléiade

Référence électronique

Isabelle Périer, "Le jeu de rôle, une autre forme de narration sérielle? », Itinéraires [En ligne], 2016-2 |

2017, mis en ligne le 25 avril 2017, consulté le 02 octobre 2020. URL : http://journals.openedition.org/ itineraires/3453 ; DOI : https://doi.org/10.4000/itineraires.3453

Ce document a été généré automatiquement le 2 octobre 2020.

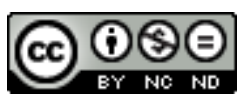

Itinéraires est mis à disposition selon les termes de la licence Creative Commons Attribution - Pas d'Utilisation Commerciale - Pas de Modification 4.0 International. 


\title{
Le jeu de rôle, une autre forme de narration sérielle?
}

\author{
Pen and Paper Roleplaying Game, Another Serial Narration?
}

\author{
Isabelle Périer
}

1 Le jeu de rôle est une pratique ludique qui a aujourd'hui plus de quarante ans mais qui demeure souvent mal connue. Elle est conviviale car elle nécessite plusieurs joueurs entre trois et six -, assis autour d'une table qui accueille le matériel nécessaire au jeu. Ces joueurs incarnent un personnage qu'ils ont eux-mêmes créé en suivant les règles du jeu ou qu'ils ont choisi dans un panel de personnages dits " pré-tirés ", c'est-à-dire déjà créés. L'un des joueurs a un rôle particulier autour de la table : on le nomme Meneur (ou Maître) de jeu ${ }^{1}$, car il doit connaître et maîtriser le monde fictionnel dans lequel les personnages des joueurs ${ }^{2}$ vont évoluer afin de pouvoir le leur décrire au fur et à mesure de leurs aventures ${ }^{3}$. Il incarne également les personnages non-joueurs ${ }^{4}$ qui peuplent cet univers et il fait jouer un scénario, à savoir une trame narrative que les joueurs vont suivre tout en la modifiant par leurs actions ${ }^{5}$.

2 La plupart des jeux de rôle utilisent des artefacts ludiques comme des dés, des cartes ou des jetons, des fiches permettant de noter les paramètres qui définissent les personnages des joueurs, et le matériel du MJ, à savoir ses notes, son scénario et le jeu de rôle auquel le groupe va jouer. Car si le jeu de rôle est une pratique ludique, il existe des jeux de rôle, à savoir des gammes d'ouvrages décrivant des univers qui sont autant d'espaces de jeu : cette distinction entre le jeu de rôle - la pratique - et les jeux de rôle - les livres-univers qui encadrent cette pratique ${ }^{6}$ - permet de pointer l'une des spécificités des jeux de rôle comme objets-livres : les livres de jeu de rôle peuvent se lire indépendamment de toute pratique, pour le plaisir de découvrir des mondes fictionnels, mais leur fonctionnement premier est celui d'un mode d'emploi - souvent fort long - d'un univers de jeu et d'un système de règles'. Toutefois, le jeu de rôle en tant que pratique n'existe que dans l'actualisation de ce mode d'emploi qui contient une infinité d'histoires possibles, dont quelques-unes seulement seront jouées autour d'une table. 
3 Dans ce cadre, nous nous proposons de montrer en quoi le jeu de rôle est une forme de narration sérielle. En effet, il s'agit d'une pratique fictionnelle qui repose, dans son fonctionnement même, qu'il soit externe et éditorial ou interne et privé, sur le principe de l'expansion. C'est ce principe d'expansion que nous allons développer en montrant en quoi il est le parent d'autres formes de sérialité et comment il s'inscrit dans la logique transmédiatique contemporaine qui favorise les narrations sérielles et leur développement dans différents médias. Il est pourtant à la fois extraordinairement fécond et mortifère, car il peut déboucher sur un chaos narratif fatal pour les mondes fictionnels, menacés de devenir informes, contradictoires ou stéréotypés. Toutefois le jeu de rôle possède des mécanismes internes propres à réguler ce principe d'expansion afin de permettre à l'univers fictif de conserver sa cohérence, son originalité et sa fécondité. Notre propos s'articulera en trois parties, qui sont autant de niveaux d'analyse possibles, en essayant de montrer à l'intérieur de ces parties comment joue cette dynamique entre limite et expansion : d'abord, le jeu de rôle en tant que média s'inscrivant dans un système transmédiatique, ensuite le jeu de rôle en tant que gamme éditoriale, enfin le jeu de rôle en tant que pratique interactive et intercréative des joueurs.

\section{Le jeu de rôle comme élément d'un système transmédiatique}

4 Le jeu de rôle peut naître en prolongement transmédiatique d'un univers fictionnel déjà développé dans un autre média (Ryan et Thon 2014). Le cas le plus fréquent est celui du prolongement d'un univers littéraire comme l'adaptation du monde fictionnel de Lovecraft dans The Call of Cthulhu (1981) ou celle du roman de Mathieu Gaborit, Les Chroniques des Crépusculaires ${ }^{8}$ dans le jeu nommé Agone (1999). Toutefois, les développements éditoriaux autour des œuvres littéraires sont souvent compliqués : en effet, le jeu The Call of Cthulhu a connu à ce jour sept éditions et plus de quatre-vingt-dix suppléments en trente ans. L'œuvre de Lovecraft a également été adaptée en un livreunivers nommé Kadath, Le Guide de la Cité Inconnue (2010), qui est lui-même à la source d'un petit jeu de rôle de six scénarios nommé Kadath (2011), lequel s'appuie très explicitement sur le livre-univers (Périer 2013). De même, l'intégrale des Royaumes Crépusculaires de Mathieu Gaborit, comprenant Les Chroniques des Crépusculaires et Abyme (1996-1997), a donné naissance au jeu de rôle Agone (2000), puis à une boîte décrivant la cité d'Abyme, proposant une demi-douzaine de scénarios, recelant ses propres règles et pouvant être considérée soit comme un jeu indépendant du jeu Agone, soit comme l'un de ses prolongements. Or, quelques années plus tard, un livre-univers nommé Abyme, le Guide de la cité des ombres (2009) a vu le jour. Il s'agit, comme pour Kadath, dont il est le devancier, du guide touristique de la ville imaginaire d'Abyme, rédigé à la fois comme un récit et comme un guide de voyage (Caïra 2014). À partir de ce guide est né un petit jeu, Abyme. Aventures dans la Cité des Ombres (2010), qui est un développement parallèle à la boîte originelle, sur le même format que le jeu Kadath. Ainsi, les limites assignées à l'expansion de mondes fictionnels préexistants sont simples : l'œuvre dont est tiré le jeu fixe le canon (Saint-Gelais 2011 : 386) qui constitue la limite de l'expansion de ces univers, même si cette limite disparaît lorsque les jeux s'engouffrent dans les zones inexplorées ou juste esquissées par la fiction de départ ${ }^{9}$. Parfois, les développements autour d'univers imaginaires sont encore bien plus compliqués et certains semblent ne 
pas rencontrer de limites. L'univers de Warhammer $40 \mathrm{~K}$ est un exemple particulièrement saillant de développement d'un univers fictionnel transmédiatique où s'entremêlent jeu de figurines, jeu de rôle, jeu vidéo, littérature et films d'animation dans un mouvement d'expansion amorcé dès 1987 et qui ne semble pas trouver de limites ${ }^{10}$.

Inversement, les univers de jeu de rôle peuvent être à l'origine d'adaptations et de développements dans d'autres médias. Par exemple, le jeu de rôle Vampire : la Mascarade (1991) a donné lieu à de nombreuses expansions, dont certaines sont devenues des jeux à part entière comme Mage: l'Ascension (1993) ou Loup-garou: l'Apocalypse (1991). Vampire : la Mascarade a également été adapté en jeu vidéo à deux reprises : Vampire : La Mascarade - Rédemption (2000) et Vampire: The Masquerade - Bloodlines (2004). Ce monde fictionnel a aussi été adapté en romans : le cycle Vampire: La Mascarade - La malédiction du sang de Gherbod Fleeming et Vampire: Le cycle de la Mort Rouge de Robert Weinberg, ainsi qu'en séries de romans développés autour de la notion de clan. Il a même donné naissance à une série télévisée, Kindred : le Clan des maudits (1996), annulée brutalement suite au décès de l'acteur jouant le personnage principal. De plus, le film nommé Underworld (2003) représente un univers fictionnel si proche de l'univers de Vampire : la Mascarade que la maison d'édition White Wolf a intenté une action en justice, trouvant plus de soixante points de recoupement ; l'affaire s'est terminée en audience privée où les deux partis ont dû trouver un terrain d'entente, au vu des développements ultérieurs du film. Dans ce cas d'un univers de jeu adapté dans d'autres médias, c'est bien l'univers de jeu qui est le canon limitant l'expansion narrative de son univers. On notera ici un renversement de légitimité : le canon est dès lors ludique et non plus littéraire, ce qui tend à reléguer les productions dérivées dans les affres de la paravoire de l'infralittérature. Toutefois, quelques efforts de renversement de légitimité ont vu le jour. Par exemple, la gamme Nephilim (1991) a connu plusieurs vagues d'adaptations littéraires : le Cycle des Phénix de Sébastien Pennes (1994-1998) et le Cycle du Chant de la Terre d'Isabelle et David Collet (1998) pour la deuxième édition ainsi que le Cycle des Déchus de Fabien Clavel pour la troisième édition, réédité dans une version augmentée en deux volumes à l'occasion de la sortie de la quatrième édition en 2012. Le visuel très travaillé de cette dernière édition tend à vouloir accroître la légitimité d'un cycle certes né d'un jeu, mais qui a toujours mis en avant des origines intertextuelles légitimes, voire prestigieuses, comme le Pendule de Foucault d'Umberto Eco. Ce mouvement de légitimation tend à s'accentuer puisque les Vélins Carminae (Périer et Weil 2013), un recueil de textes subjectifs des précédentes éditions enrichi de poèmes originaux, a vu le jour, avec pour objectif de décrire le monde de Nephilim de l'intérieur, c'est-à-dire subjectivement et poétiquement. Cette tentative d'adaptation poétique d'un univers ludique marque la progression d'une certaine légitimation. Le jeu de rôle participe ainsi à l'expansion transmédiatique des mondes fictionnels comme beaucoup d'autres médias ${ }^{11}$.

\section{Le jeu de rôle comme gamme éditoriale}

Si le jeu de rôle est un média possible au sein d'un complexe transmédiatique se rapportant à un monde de fiction, il est, dans ses principes mêmes, voué à l'expansion d'univers. En effet, une gamme traditionnelle de jeu de rôle ${ }^{12}$ se décompose en plusieurs unités éditoriales qui sont le développement de différents aspects du jeu et de son univers. 
7 Le livre de base est normalement le premier livre de la gamme à sortir car il contient tous les fondamentaux nécessaires pour jouer : l'exposition du monde fictionnel dans ses grandes lignes, la création de personnage, les règles, des conseils pour le $\mathrm{MJ}$ et éventuellement un premier scénario dit «d'introduction». Par la masse des informations à réunir, c'est souvent le plus gros livre de la gamme. Il se divise parfois en deux opus: le livre des joueurs, qui contient un exposé condensé du monde, la création de personnage et les règles, et le livre du meneur, qui contient un exposé bien plus dense du monde, ses secrets, les conseils donnés au $\mathrm{MJ}$, quelques règles optionnelles, un premier survol des antagonistes et le scénario d'introduction. Ce ou ces livres sont donc la matrice du jeu, en ce qu'ils en posent les prémisses, et le canon premier puisqu'ils posent la «norme » sur laquelle les auteurs ultérieurs et les joueurs vont s'appuyer pour développer le monde fictionnel.

8 La parution du livre de base est généralement suivie de celle de l'écran, également nommé « paravent » : un morceau de carton en trois ou quatre pans, illustré et solide, qui permet au MJ de dissimuler son matériel. L'écran a également pour fonction d'annoncer le visuel du jeu: c'est l'illustration que les joueurs regarderont le plus souvent, avec la couverture du livre de base. Elle doit donc être particulièrement soignée car elle est le reflet du jeu. Il est souvent vendu avec un scénario et/ou quelques développements sur l'univers, mais il est également important car il est souvent couplé avec une carte de grand format de l'univers ludique. Or cette carte, outre son importance dans la pratique ludique des joueurs, est capitale pour plusieurs raisons. Elle est d'abord un objet de rêverie par ses contours, son onomastique et les multiples histoires qu'elle recèle en germes. La rêverie des auteurs et des joueurs de jeu de rôle est en quelque sorte une rêverie sur la carte d'un monde imaginaire : par références, conscientes ou inconscientes aux codes du genre et à une forme de mimèsis intertextuelle, elle donne l'impression de receler une multitude de récits potentiels, qui sont d'autant plus séduisants qu'ils resteront, pour la plupart, à l'état de possibles narratifs ${ }^{13}$. De plus, cette carte est une structure, une grille qui pose le cadre des développements futurs de l'univers: chaque compartimentage, planète, continent, pays, contrée, etc. est une petite case que les inventions des auteurs de la gamme ou des joueurs vont venir remplir. Elle est, en quelque sorte, la représentation cartographique du travail imaginaire à accomplir. Enfin, elle est aussi la limite posée à l'imaginaire : la carte du monde fictionnel définit le cadre de l'expansion de la fiction. Elle est l'une des limites permettant à ce monde de garder sa cohérence.

9 Une gamme propose également des suppléments que l'on peut classer en trois catégories : les suppléments de règles, les suppléments d'univers et les scénarios. Les suppléments de règles peuvent proposer des modifications ou des options supplémentaires à la création de personnage, permettant ainsi d'augmenter la puissance des PJ mais également d'étoffer leur histoire personnelle, agissant ainsi comme une matrice de prequel ${ }^{14}$ à leur histoire. Ils fonctionnent donc aussi comme des générateurs d'histoires au sens où ils permettent l'expansion de la biographie des personnages. Ils peuvent également proposer de nouvelles règles pour gérer des situations particulières : par exemple le supplément L'Art de la guerre (2006) pour le jeu Qin (2005) permettant de jouer des héros de film de sabre au moment de la création de l'Empire du Qin propose et développe une armature de règles afin de simuler de manière à la fois rationnelle et cinématographique de grandes scènes de batailles. Ces armatures de règles permettent d'engendrer de la narration en créant de nouvelles 
structures ludo-narratives (Guardiola 2005 : 161-174). Enfin, ces suppléments de règles permettent d'ouvrir de nouvelles possibilités aux joueurs en développant de nouveaux éléments pour leurs personnages, comme des magies ou des voies spirituelles. Là encore, ils créent un espace de règles fertile en narration puisqu'ils ouvrent de nouveaux possibles narratifs aux joueurs et au MJ.

Si les suppléments de règles sont fertiles en développements narratifs, il est bien évident que ce sont les suppléments voués à l'expansion du monde fictionnel qui sont les plus féconds. Ces derniers développent l'univers dans trois directions, qui sont les trois éléments indispensables à toute narration : l'espace, le temps, les personnages. Certains suppléments sont voués à l'exploration de l'espace de l'univers fictionnel : ils sont, en quelque sorte, le développement de la grille que constitue la carte ${ }^{15}$. Ils décrivent ainsi des continents, des pays ou de simples villes en multipliant les points de vue et les méthodes : économie, géographie, population, us et coutumes, etc. Ces textes oscillent entre la description objective et omnisciente au présent et la description ou narration subjective, qui développe un point de vue particulier sur le monde et lui donne davantage de relief. D'autres suppléments explorent le monde fictionnel dans son histoire ou ses mythes, lui conférant ainsi une profondeur particulière puisque temporelle ${ }^{16}$. Enfin, beaucoup de suppléments sont voués à développer les organisations ou les personnages auxquels sont confrontés les joueurs. Bien évidemment, aucun $\mathrm{MJ}$ ne se servira de l'intégralité de toutes ces expansions fictionnelles au cours de sa pratique : elles sont une manière de nourrir sa rêverie afin de l'aider à construire ses propres histoires. Mais elles sont également le moyen par lequel le monde fictionnel se densifie et atteint un niveau de détail tel qu'il en devient réaliste, selon le principe de l'effet de réel de Barthes (1982) : plus un MJ est en mesure de peupler son univers fictionnel de détails n'ayant rien à voir avec l'intrigue du scénario, plus cet univers fictionnel acquerra une existence autonome et réaliste pour les joueurs.

11 Enfin, une gamme de jeu de rôle s'articule bien souvent également en scénarios. Ces derniers sont de deux sortes : les one-shot, comme leur nom l'indique, se jouent en une ou deux séances et sont indépendants les uns des autres. Ils relèvent plutôt de l'expansion sérielle au sens que peut lui donner Anne Besson (2004: 15). À l'inverse, les campagnes représentent une longue aventure ou plutôt un cycle d'aventures liées entre elles; feuilletonnantes, elles relèvent de l'expansion cyclique (Ibid.) en ce qu'elles sont divisées en épisodes qui ont un début, un milieu et une fin mais qui constituent aussi une méta-intrigue globale. Le principe des campagnes est aussi vieux que celui du jeu de rôle, ou presque : l'une des campagnes les plus célèbres de The Call of Cthulhu, Les Masques de Nyarlathotep a vu le jour en 1984. Toutefois, les gammes de jeu de rôle ont eu tendance à devenir de plus en plus feuilletonnantes à partir des années 1990, dans un mouvement parallèle à celui de la littérature, du cinéma ou des séries télévisées. Ainsi, la gamme COPS (2003), où les joueurs incarnent des agents d'élite d'un service de police nommé COPS à Los Angeles en 2030, est conçue sur le modèle d'une série télévisée : les suppléments déroulent une storyline qui s'étend sur quelques mois à chaque fois et sont répartis en quatre saisons. Le jeu s'appuie donc sur un monde fictionnel très dynamique qui se modifie à chaque supplément. Sans aller jusque-là, des gammes comme Wasteland, les terres gâchées (2011) sont conçues pour que les groupes jouent les scénarios et les volumes de la campagne publiée dans l'ordre, déroulant ainsi le fil d'une méta-intrigue qui constitue le cœur du jeu. Cette méta-intrigue permet, comme a pu le faire COPS, de rapprocher la structure d'une gamme de jeu de rôle de celle d'une 
série : chaque scénario est assimilable à un épisode, une suite de scénarios peut former un arc narratif ${ }^{17}$, une campagne est l'équivalent d'une saison, l'intégralité de l'histoire des joueurs est alors la méta-intrigue de la série (Newman 2006). Certaines productions tentent même de se caler sur le temps historique afin de renforcer l'effet de réel et de sérialité : la campagne des Chroniques de l'Apocalypse (1999-2000) pour la seconde édition de Nephilim est divisée en cinq volumes qui sont parus tout au long de l'année 2000, tous les deux mois environ, laissant la possibilité aux tables de la jouer de manière régulière tout au long de l'année, à la manière d'une série télévisée.

12 Est-ce à dire qu'une gamme de jeu de rôle peut connaître une expansion infinie? Théoriquement, oui, dans la mesure où la description d'un monde fictionnel est une tâche infinie ${ }^{18}$. Pragmatiquement, non. D'une part, les finances des joueurs n'y résisteraient pas. D'autre part, une gamme de jeu de rôle est tenue de respecter un canon exigeant : le canon de l'œuvre originale, si le jeu est une adaptation, mais surtout l'intégralité des écrits de la gamme précédant le volume en écriture. Or, si le canon est feuilletonnant, les auteurs se doivent de suivre la storyline développée par la gamme; mais les joueurs ne suivent pas nécessairement toute la gamme et ils n'ont pas forcément tout joué. Ainsi, la multiplication des suppléments multiplie les risques d'écart entre le monde fictionnel des joueurs et celui de la gamme officielle, et donc les risques de décrochement. En effet, à quoi bon acheter des suppléments décrivant un univers qui n'a plus qu'un rapport lointain avec celui qui a été construit avec ses joueurs autour de la table de jeu? La mémoire est également une limitation pragmatique à l'expansion des univers de fiction. D'une part, la multiplication des informations rend la tâche des auteurs et de l'éditeur de plus en plus ardue au fur et à mesure du développement de la gamme - à supposer que le personnel éditorial ne change pas, ce qui est fort rare - et multiplie les risques d'incohérences. D'autre part, les lecteurs s'y retrouvent de plus en plus difficilement : le risque de décrochement de la gamme officielle s'accroît une fois encore devant la difficulté pour les utilisateurs de maitriser toutes les informations liées au monde fictionnel ${ }^{19}$. Enfin, les gammes aux innombrables suppléments sont également un repoussoir pour tous ceux qui sont intéressés par le jeu ou l'univers fictionnel, mais qui, arrivés en cours de route, doivent trouver des suppléments épuisés, lire des centaines de pages pour combler leur retard et dépenser des sommes colossales pour reconstituer toute la collection.

13 Ainsi, devant le risque d'expansion illimitée de la gamme, les éditeurs de jeux de rôle ont entériné une pratique répandue en littérature mais indispensable dans le jeu: les nouvelles éditions. Celles-ci permettent de corriger certaines faiblesses, notamment dans les règles, ou de les modifier pour suivre l'évolution globale du monde du jeu de rôle. Mais leur grand avantage consiste surtout à opérer une mise à jour dans la storyline globale du monde, de résumer ce qui s'est passé dans les suppléments précédents et de repartir sur de nouvelles bases. Chaque nouvelle édition est donc à la fois un renouveau - des règles et de certains pans du monde fictionnel - et une continuation permettant de donner un nouveau souffle au jeu en conservant les anciens joueurs et en espérant en attirer de nouveaux. Ainsi, la gamme de Nephilim seconde édition était devenue si touffue que des incohérences avaient fini par se glisser dans l'univers fictionnel. Un grand chantier a été mis en place autour des Chroniques de l'Apocalypse en 1999 et en 2000, puisqu'un supplément nommé Testament a vu le jour en 1999, qui faisait le point sur la storyline et les plans des opposants aux Nephilim, les Arcanes mineurs. Cette mise au point permettait à chaque table de commencer la campagne des Chroniques de l'Apocalypse en partant des mêmes informations. La fin de la 
campagne a débouché sur une révélation - comme l'annonçait son titre - ouvrant sur la troisième édition du jeu, Nephilim: Révélation en 2002. Au début des années 2000, l'éditeur White Wolf a fait un choix encore plus radical : devant l'ampleur des gammes de Vampire, Mage et Loup-Garou, il a décrété que l'Apocalypse des années 2000, tant attendue et tant invoquée tout au long de ces différentes gammes, allait avoir lieu. Pour chaque gamme, il a sorti une grande campagne permettant aux tables de mettre fin à l'histoire en proposant des fins du monde différentes selon les jeux. Par exemple, il a sorti la campagne Géhenne (2004) pour Vampire: La Mascarade qui propose quatre fins du monde, de la plus intimiste (un huis clos) à la plus spectaculaire (la destruction du monde et sa rédemption possible par les PJ). Puis, il a effectué un reboot, en lançant une nouvelle gamme nommée Le Monde des Ténèbres (2004) se déclinant en différentes races jouables constituant autant d'arcs possibles pour appréhender ce nouveau monde fictionnel : Vampires, Mages, Loups-garous, etc. Il s'agit bien d'un reboot, car le monde fictionnel est une déclinaison du monde des ténèbres antérieur sans être le même, et il bénéficie surtout d'une cohérence renforcée puisque toutes ses déclinaisons ont été pensées en même temps, contrairement à l'univers précédent qui s'est peuplé petit à petit, par expansions progressives. Ainsi, devant l'expansion proliférante du premier monde des ténèbres, générateur d'incohérences, de redites et de paradoxes, White Wolf a choisi le reboot, proposant un nouvel univers à développer, en partant de prémisses plus saines car d'emblée prévues.

\section{Le jeu de rôle comme pratique}

Enfin, le jeu de rôle est surtout une pratique narrative et ludique fonctionnant comme une expansion fictionnelle interactive et intercréative (David 2015). D'abord, tout comme une gamme de jeu de rôle peut prévoir des scénarios one-shot ou des campagnes, le MJ peut mener des parties one-shot ou des campagnes. La partie one-shot se déroule en une séance, avec des personnages conçus par les joueurs ou des pré-tirés, c'est-à-dire des personnages déjà préparés soit par l'éditeur soit par le MJ pour les besoins spécifiques du scénario. Le one-shot se joue donc sur une courte durée et propose une histoire qui n'aura probablement pas de suite. En revanche la campagne, qui est le format favori des groupes de joueurs fixes et assidus, se joue sur le long terme car elle repose sur une méta-intrigue dont le noyau n'est autre que le groupe de joueurs. Certes, il peut y avoir des absents, des disparitions de PJ pour des raisons externes dues à la vie réelle, des PJ morts qui seront remplacés, mais le cœur de la campagne reste bien le groupe des personnages et leurs aventures. Cette entité du groupe est même souvent prévue par certains jeux: dans Agone, les personnages sont des Inspirés luttant contre le Masque et ils peuvent se regrouper en un Domaine, une sorte de base stratégique qu'ils ont à gérer. Le groupe de PJ va donc vivre des aventures dans un monde fictionnel : ces aventures peuvent être rédigées par les auteurs officiels de la gamme, mais elles peuvent également avoir été écrites par le MJ ou provenir de la communauté des internautes jouant au même jeu, puisque l'activité rôliste est très vive sur le Web et permet l'échange d'informations et de scénario ${ }^{20}$. Or, pour chaque table, le monde fictionnel ainsi déroulé sera différent. En effet, même si le groupe ne joue que les scénarios proposés par l'éditeur, il ne jouera peut-être pas tout, ou pas dans l'ordre chronologique de parution ou du recueil. Et bien évidemment, les productions du MJ ou 
d'autres MJ sont également un moteur d'expansion de la fiction puisqu'ils s'ancrent dans l'univers fictif en le prolongeant, continuant ainsi l'édifice conçu par l'éditeur.

En outre, le principe même du jeu en campagne relève de l'expansion: il constitue l'expansion de l'histoire individuelle des PJ et de celle du groupe. Chaque séance de jeu offre la possibilité pour les joueurs d'ajouter des éléments à l'histoire de leur personnage : des éléments passés inventés sur le vif, pour rire, faire rêver ou expliquer la réaction de leur personnage, une histoire d'amour impromptue nouée avec un personnage non joueur (PNJ) ou un PJ, l'adoption d'un PNJ dans le groupe en tant que serviteur, enfant ou disciple, la perte d'un être cher, etc. La dimension improvisée du jeu de rôle permet de créer de la fiction «sur le vif » et de faire évoluer l'univers fictionnel dans un sens que rien ne laissait prévoir.

Mais, plus généralement, qu'il s'agisse de jeu en campagne ou en one-shot, l'expansion est le principe même du jeu de rôle. En effet, les productions écrites du jeu de rôle, qu'il s'agisse de la gamme officielle ou des productions du MJ, fonctionnent comme la « machine paresseuse " que pointait Umberto Eco dans Lector in fabula ([1979] 1985). Elles ont besoin des joueurs et du cadre de la partie pour être actualisées car elles ne sont, en réalité, qu'un mode d'emploi. C'est donc autour de la table de jeu que la «machine paresseuse » des textes va être actualisée par l'activité non seulement du MJ, mais également des PJ, qui vont chacun imaginer les scènes décrites et peupler le monde ludique de détails qu'ils vont partager, étoffant ainsi l'univers fictionnel de détails propres à la table où se déroule la partie (David 2015). Nous pouvons prendre un exemple concret, qui constitue un topos du jeu de rôle médiéval-fantastique : la scène d'exposition du scénario commençant dans une auberge. Les PJ sont attablés à l'auberge et attendent quelqu'un. C'est là que commence le processus d'actualisation et d'expansion : quelle auberge? Dans quelle ville? Quel est son nom ? À quoi ressemblet-elle? Quel temps fait-il dehors? Autant de questions auxquelles le scénario répond probablement. La serveuse est-elle jolie ? Y a-t-il du monde? Des gens qui jouent aux cartes? Que sert-on à boire dans cette auberge? Voilà des questions plus délicates auxquelles le MJ va apporter des réponses qu'il va devoir probablement improviser. Puis-je tenter de séduire la serveuse ? Mon voisin de table a-t-il des objets de valeur sur lui ? Y a-t-il des musiciens ? Que jouent-ils? Le spectacle est-il de qualité ? Par leurs simples questions et aspirations, les PJ peuplent l'univers fictionnel de figurants et de détails qu'aucun scénario ne peut prévoir et qui peuvent devenir des personnages ou des éléments importants pour la suite de l'histoire. Cette expansion fictionnelle proliférante ${ }^{21}$ repose sur les trois éléments indispensables à tout récit que nous avons déjà rencontrés : la description des lieux et de l'espace, la narration du passé - qu'il s'agisse de celui des PJ, de PNJ ou de l'histoire du monde fictif - et l'invention de personnages, les PNJ. Ainsi, une partie de jeu de rôle ressemble en quelque sorte à une improvisation à la fois théâtrale et romanesque, un Maelstrom ${ }^{22}$, où chacun brode sur le canevas proposé par le MJ - qu'il soit officiel ou non - et sur les inventions des autres joueurs.

Devant cette dimension expansionniste, on peut être pris de vertige: si tout est possible et si l'expansion est infinie, le jeu ne risque-t-il pas d'être atteint de prolifération chaotique, ce qui finirait par le dissoudre? Pour parer à ce risque inhérent à l'expansion, le jeu de rôle possède des garde-fous qui permettent de contenir la fiction dans un cadre plus ou moins souple. Le premier garde-fou est celui des règles : les règles définissent le référentiel de ce qu'il est possible ou impossible de faire. Par 
exemple, The Call of Cthulhu permet d'incarner des investigateurs humains plus ou moins doués qui peuvent tenter de manipuler la magie du monde à leurs risques et périls. Les possibles narratifs qui s'offrent à eux outrepassent rarement ceux d'un être humain normal, même un peu doué. En revanche, Vampire: La Mascarade permet aux joueurs d'incarner un vampire, c'est-à-dire une créature immortelle dotée de pouvoirs souvent impressionnants aux yeux des humains. Toutefois, ces vampires doivent se nourrir de sang et ne peuvent sortir le jour, sous peine de se consumer. Ici, le cadre des possibles narratifs est très différent de celui qu'offre l'existence d'un humain normal: les PJ doivent chasser pour se nourrir, ils ne peuvent sortir le jour et possèdent des pouvoirs surnaturels. Ils sont à la fois plus puissants et plus vulnérables que les humains normaux. Ainsi, dans ce référentiel, les PJ ne peuvent pas décider tout d'un coup de sortir le jour pour aller au zoo: les règles leur permettront tout au plus de traverser la rue avant d'être consumés par la lumière du Soleil. Le deuxième garde-fou est le $\mathrm{MJ}$ : il maîtrise les règles, il a lu tout ou partie de la description du monde fictionnel, il a en tête l'intrigue du scénario et la méta-intrigue dans laquelle il s'inscrit et, par sa position même, il est l'arbitre décidant de ce qui est ou n'est pas possible. Enfin, le troisième garde-fou est une sorte de consensus que l'on peut nommer le pacte ou le contrat rôlistique ${ }^{23}$. Conçu sur l'idée du pacte de lecture et du pacte autobiographique de Philippe Lejeune, il s'agit de l'accord, tacite ou non, qui existe entre les joueurs et le MJ sur le fait que le groupe est réuni pour jouer à un jeu, qui se définit par une certaine ambiance, et mener à bien le scénario proposé par le MJ. Le pacte rôlistique fonctionne ainsi sur différents niveaux : il présuppose que les joueurs et le MJ sont d'accord pour jouer à un jeu particulier et qu'ils acceptent ses règles, et donc les possibles narratifs qu'elles impliquent. Il assure également l'accord de tous sur l'ambiance ou le registre donné aux parties - intimiste, épique, réaliste, les déclinaisons possibles sont nombreuses. Il implique que tous les joueurs présents acceptent de jouer le scénario proposé par le MJ, à la fois pour respecter son travail et pour assurer à tous un plaisir et une qualité de jeu optimum. Enfin, le pacte rôlistique fonctionne comme une communauté interprétative (Saint-Gelais 2011) restreinte qui permet de valider les expansions de l'univers fictif proposé. Ainsi, c'est bien le consensus dégagé en cours de partie qui permet de valider les inventions des autres joueurs, celles du MJ mais également les données de la gamme éditoriale. Ce consensus sur l'univers fictif peut se régler sur plusieurs types de normes : le canon de l'œuvre-source (en cas d'adaptation d'une œuvre littéraire ou audiovisuelle), le canon défini par la gamme de l'éditeur, ou tout simplement les goûts des joueurs autour de la table.

Ainsi, le jeu de rôle pourrait être caractérisé comme une « fiction quantique » selon les termes d'Alexis Blanchet qui la définit ainsi, dans le cadre de référence du jeu vidéo :

Chaque joueur à son niveau, par sa pratique personnelle, multiplie les états de la fiction [vidéoludique] et dévoile les univers parallèles de celle-ci. Une communauté de joueurs s'adonnant à un même titre produit donc, par ses échecs et ses réussites, une infinité d'états et de mondes possibles d'une même fiction [vidéoludique]. (Blanchet 2009 : 493)

19 Les joueurs de jeu de rôle circulent ainsi entre une infinité de mondes possibles ${ }^{24}$ déclinant un univers de référence fixé par le canon. En effet, un même MJ peut faire jouer plusieurs fois le même scénario ou la même campagne qui donnera à chaque fois lieu à une narration différente et donc à un monde possible différent. C'est sur ce vertige que repose une grande partie de la dimension sociale du jeu de rôle : comparer des expériences de jeu permet à chaque fois d'explorer l'un des mondes possibles 
déclinant «la machine paresseuse » du scénario. Chaque jeu de rôle est en quelque sorte un «multivers » dont le groupe de joueurs n'explore qu'une déclinaison.

Ainsi, le jeu de rôle est bien une narration sérielle fonctionnant par essence sur le principe de l'expansion, et ce sur trois niveaux : un niveau transmédiatique, un niveau éditorial, un niveau ludique. Le jeu de rôle met donc en jeu la possibilité d'une expansion infinie d'un univers fictionnel donné et pose la question des limites de cette expansion. Par-delà les limites qui ont pu être mentionnées, nous insisterons sur la notion de pacte ou de contrat rôlistique qui fait du groupe une communauté interprétative à même de décider du respect ou du non-respect du canon, qu'il provienne d'une œuvre originale ou de la gamme elle-même. Car le jeu de rôle est avant tout une pratique fictionnelle et ludique conviviale et intercréative qui permet de créer ensemble de la fiction.

\section{BIBLIOGRAPHIE}

Barthes, Roland, 1982, «L'effet de réel », dans G. Genette et T. Todorov (dir.), Littérature et réalité, Paris, Seuil, p. 81-90.

Bayard, Pierre, 2012, Comment parler des livres que l'on n'a pas lus ?, Paris, Minuit.

Besson, Anne, 2004, D'Asimov à Tolkien. Cycles et séries dans la littérature de genre, Paris, CNRS Éditions.

Besson, Anne, 2015, Constellations. Des mondes fictionnels dans l'imaginaire contemporain, Paris, CNRS Éditions.

Besson, Anne, Prince, Nathalie et Bazin, Laurent (dir.), 2016, Mondes fictionnels, mondes numériques, mondes possibles, Rennes, PUR.

Blanchet, Alexis, 2009, Les Synergies entre cinéma et jeu vidéo : histoire, économie et théorie de l'adaptation vidéoludique, thèse de doctorat, Université Paris Ouest Nanterre La Défense.

Briand, Romaric, 2014, Le Maelstrom, Édition indépendante, p. 239-282, http://

leblogdesens.blogspot.fr/2014/11/le-maelstrom-est-sorti.html, consulté le 9 février 2017.

Caïra, Olivier, 2007, Jeux de rôle, Les forges de la fiction, Paris, CNRS Éditions.

Caïra, Olivier, 2014, « Ourobores. Explorer les virtualités des mondes fictionnels après le tournant diégétique », Revue critique de fixxion française contemporaine, $\mathrm{n}^{\circ}$ 9, p. 72-86, [En ligne], http://

www.revue-critique-de-fixxion-francaise-contemporaine.org/rcffc/article/view/fx09.07, consulté le 10 juin 2015.

David, Coralie, 2015, Le Jeu de rôle sur table : l'intercréativité de la fiction littéraire, thèse de doctorat, Université Paris 13.

Eco, Umberto, [1979] 1985, Lector in fabula, trad. M. Bouzaher, Paris, Le Livre de Poche.

Guardiola, Emmanuel, 2005, « L'histoire que nous faisons vivre au joueur : la structure ludonarrative », dans S. Genvo (dir.), Le game design de jeux vidéo. Approches de l'expression vidéoludique, Paris, L'Harmattan, p. 161-174. 
Hamon, Philippe, 1981, Introduction à l'analyse du descriptif, Paris, Hachette.

Jenkins, Henry, 2006, Convergence Culture: Where Old and New Media Collide, New York, New York University Press.

Klastrup, Lisbeth et Tosca, Susana, 2004, « Transmedial Worlds - Rethinking Cyberworld Design », dans 2004 International Conference on Cyberworlds, p. 409-416, [En ligne], http:// www.itu.dk/people/klastrup/klastruptosca_transworlds.pdf, consulté le 18 juin 2014. DOI : 10.1109/CW.2004.67.

Letourneux, Mathieu, 2005, «Les univers de fiction des jeux vidéo », dans S. Genvo (dir.), Le game design de jeux vidéo. Approches de l'expression vidéoludique, Paris, L'Harmattan.

Newman, Michael Z., 2006, « From Beats to Arcs: Toward a Poetics of Television Narrative », The Velvet Light Trap, $\mathrm{n}^{\circ}$ 58, p. 16-28.

Parker, Felan, 2008, « The Significance of Jeep Tag. On Player-Imposed Rules in Video Games », Loading..., vol. 2, $\mathrm{n}^{\circ}$ 3, [En ligne], http://journals.sfu.ca/loading/index.php/loading/article/view/ $44 / 41$.

Pavel, Thomas, 1988, Univers de la fiction, Paris, Seuil.

Périer, Isabelle, 2013, « Adaptation et transmédialité : Kadath la Cité Inconnue », communication au colloque « Présence de Lovecraft : l'illustration en question » (Clermont-Ferrand, 13 et 14 juin 2013), organisé par C. Gelly et G. Menegaldo.

Périer, Isabelle, 2015, « Le livre de base de jeu de rôle : du mode d'emploi au support de rêverie », communication au colloque « Les Quarante ans du jeu de rôle » (Universités Paris 3 et Paris 13, 12-14 juin 2015), organisé par D. André, V. Berry, C. Cornillon, I. Périer et A. Quadrat.

Périer, Isabelle, 2016, « De la fiction romanesque au monde ludique », dans A. Besson, N. Prince et L. Bazin (dir.), Mondes fictionnels, mondes numériques, mondes possibles, Rennes, PUR, p. 185-200.

Périer, Isabelle, 2017, « Lecteurs, joueurs, scripteurs, auteurs : comment relativiser certaines notions », Revue française des sciences de l'information et de la communication, $\mathrm{n}^{\circ} 10$, [En ligne], http://rfsic.revues.org/2857

DOI : $10.4000 /$ rfsic. 2857

Ryan, Marie-Laure, 1992, Possible Worlds, Artificial Intelligence and Narrative Theory, Bloomington, Indiana University Press.

Ryan, Marie-Laure et Thon, Jan-Noël (dir.), 2014, Storyworlds across Media, Lincoln, University of Nebraska Press.

Saint-Gelais, Richard, 2011, Fictions transfuges. La transfictionnalité et ses enjeux, Paris, Seuil.

Salen, Katie et Zimmerman, Eric, 2004, Rules of Play: Game Design Fundamentals, Cambridge, MA, MIT Press.

Schaeffer, Jean-Marie, 1999, Pourquoi la fiction?, Paris, Seuil.

Schmoll, Patrick, 2013, « Relire Jacques Henriot à l'ère de la société ludique et des jeux vidéo », Sciences du jeu, $\mathrm{n}^{\circ} 1$, [En ligne], http://sdj.revues.org/271

DOI : $10.4000 /$ sdj.271

Sepulchre, Sarah (dir.), 2011, Décoder les séries télévisées, Bruxelles, De Boeck. 


\section{Corpus}

\section{Jeux de rôle}

Abyme, 2000, Multisim.

Agone, 1999, Multisim.

COPS, 2003, Asmodée.

Géhenne, 2004, White Wolf.

Kadath, Aventures dans la Cité Inconnue, 2011, Les XII Singes.

L'Art de la guerre, 2006, $7^{\mathrm{e}}$ Cercle.

Le Monde des Ténèbres, 2004, White Wolf.

Les Chroniques de l'Apocalypse, 1999-2000, Multisim.

Les Masques de Nyarlathotep, 1984, Chaosium.

Loup-garou : L'Apocalypse, 1991, White Wolf Publishing.

Mage : L'Ascension, 1993, White Wolf Publishing.

Nephilim, 1991, Multisim.

Qin, 2005, $7^{\mathrm{e}}$ Cercle.

Testament, 1999, Multisim.

The Call of Cthulhu, 1981, Chaosium.

Vampire: La Mascarade, 1991, White Wolf Publishing.

Wasteland, les terres gâchées, 2011, Département des Sombres Projets.

\section{Romans et textes littéraires}

Abyme, Le Guide de la Cité des Ombres, 2009, Saint-Laurent-d'Oingt, Mnémos.

Clavel, Fabien, 2012, Les Déchus, Saint-Laurent-d'Oingt, Mnémos.

Collet, Isabelle et Collet, David, 1998, Le Cycle du Chant de la Terre, Saint-Laurent-d'Oingt, Mnémos. Gaborit, Mathieu, 1996-1997, Abyme, Saint-Laurent-d'Oingt, Mnémos.

Gaborit, Mathieu, 1995-1996, Les Chroniques des Crépusculaires, Saint-Laurent-d'Oingt, Mnémos.

Kadath, Le Guide de La Cité Inconnue, 2010, Saint-Laurent-d'Oingt, Mnémos.

Pennes, Sébastien, 1994-1998, Le Cycle des Phénix, Saint-Laurent-d'Oingt, Mnémos.

Périer, Isabelle et Weil, Frédéric, 2013, Les Vélins Carminae, Saint-Laurent-d'Oingt, Mnémos.

\section{Films et séries}

Leekley, John, 1996, Kindred : Le Clan des maudits, FOX.

Wiseman, Len, 2003, Underworld, Lakeshore Entertainment. 


\section{Jeux vidéo}

Vampire : La Mascarade - Rédemption, 2000, Activision Blizzard.

Vampire : The Masquerade - Bloodlines, 2004, Activision Blizzard.

\section{NOTES}

1. Abrégé en MJ dans la suite de cet article.

2. Abrégé en PJ dans la suite de cet article.

3. Il existe également des jeux dits à «narration partagée » où le rôle de meneur de jeu n'existe pas en tant que tel car son autorité sur la fiction est partagée entre tous les joueurs. Voir "Autorité partagée/narration partagée », Les Ateliers imaginaire, [En ligne], http:// lesateliersimaginaires.com/glossaire/

autorit\%C3\%A9_partag\%C3\%A9e_narration_partag\%C3\%A9e (consulté le 9 février 2017).

4. Abrégés en PNJ dans la suite de cet article.

5. Pour plus de détails, voir Olivier Caïra (2007: 15 et suiv.).

6. Cette distinction entre le "game " (l'objet-jeu) et le « play " (l'expérience de jeu) n'est pas une spécificité du jeu de rôle mais une propriété du jeu en général. Pour une mise au point claire sur ces concepts, voir Patrick Schmoll (2013).

7. Sur le livre de jeu de rôle comme mode d'emploi, voir Isabelle Périer (2015).

8. Le cycle des crépusculaires est une trilogie publiée aux éditions Mnémos et composée de trois romans : Souffre-Jour (1995), Les Danseurs de Lorgol (1996) et Agone (1996).

9. Sur l'adaptation d'œuvres romanesques en mondes ludiques, voir Isabelle Périer (2016).

10. Pour se rendre compte de cette expansion, voir "Warhammer 40,000", Wikipédia, l'encyclopédie libre, 3 avr 2017, http://fr.wikipedia.org/w/index.php? title=Warhammer_40,000\&oldid=136061548 (consulté le 10 février 2017).

11. Sur la notion d'inflation des mondes fictionnels, voir Anne Besson (2015).

12. Nous laisserons de côté tous les jeux de rôle qui ne proposent que quelques aventures indépendantes à jouer, c'est-à-dire les " petits formats ».

13. On pourrait parler de «narrativité latente » ou de «narrativité diffuse » comme peut le faire Mathieu Letourneux (2005: 195 et 203).

14. "Par opposition à "sequel", "suite", et donc épisode d'un ensemble qui constitue le prolongement de ses prédécesseurs à la fois dans la chronologie de parution et dans celle de l'intrigue, le néologisme "prequel" désigne un "devancement". Postérieur dans la chronologie de parution, il n'en rapporte pas moins une phase antérieure du développement de l'intrigue. Genette a proposé 1[a] catégorie de "continuation analeptique" (prequel) pour souligner ce procédé aussi ancien que la forme cyclique elle-même [...]. » (Besson 2004 : 242)

15. C'est ce que Klastrup et Tosca (2004) nomment le topos.

16. C'est ce que Klastrup et Tosca (2004) nomment le mythos.

17. «Par arc narratif, on entend un scénario particulier qui suit le modèle dramaturgique classique où l'action se construit progressivement jusqu'à arriver à son paroxysme au moment du dénouement. Dans une série à épisodes, l'arc est intentionnellement prolongé au-delà d'un épisode donné de manière à inciter les téléspectateurs à revenir pour découvrir le dénouement dans un épisode ultérieur. » (Sepulchre $2011: 189$ )

18. On peut relier cette tâche infinie à «la liberté incontrôlable du descriptif » qu'évoque Philippe Hamon dans son Introduction à l'analyse du descriptif (1981 : 15). 
19. Les réflexions de Pierre Bayard (2012) sur les limites de la mémoire du lecteur de littérature peuvent pleinement s'appliquer à la lecture des ouvrages décrivant un monde fictionnel de jeu de rôle.

20. Le jeu de rôle est un média appartenant au modèle collaboratif développé par Henry Jenkins (2006 : 136). Voir aussi Périer (2017).

21. Cette caractéristique du jeu de rôle est à rapprocher de la notion de " gameplay expansif » telle que peut la définir Felan Parker (2008).

22. Sur la notion de Maelstrom en jeu de rôle, voir Romaric Briand (2014 : 239-282).

23. Ce terme de contrat rôlistique revient souvent dans la communauté, par exemple sur le site Cosmo Ørbüs qui tente de le définir: http://saint-epondyle.net/blog/jeu-de-role/le-contratrolistique/ (consulté le 9 février 2017). On peut le rapprocher de la notion de « règles implicites » développée par Salen et Zimmerman (2004:130).

24. Si la notion de monde possible en littérature appelle de nombreuses références comme Pavel (1988), Schaeffer (1999), Ryan (1992), son prolongement dans les univers ludiques a été exposé et théorisé dans l'introduction d'Anne Besson à l'ouvrage collectif Mondes fictionnels, mondes numériques, mondes possibles (Besson, Prince et Bazin 2016).

\section{RÉSUMÉS}

Après avoir présenté le jeu de rôle (JdR) sur table, cet article a pour but d'analyser son fonctionnement en tant que narration sérielle. En effet, le JdR propose d'explorer des mondes fictionnels par le truchement de narrations fonctionnant sur le principe de l'expansion, principe qui peut se montrer aussi fertile que chaotique dans la mesure où, avec le temps et la pratique, il peut détruire la cohérence et la vraisemblance de ces mondes fictionnels. Toutefois, ce principe d'expansion peut être régulé de diverses manières. C'est cette dialectique entre expansion et régulation que nous nous proposons d'étudier. Pour cela, nous adopterons trois points de vue : le JdR en tant que média fonctionnant dans un système transmédiatique, le JdR en tant que gamme d'ouvrages et le JdR en tant que pratique.

After a presentation of what is "pen and paper" role-playing game (RPG), this article aims to analyze how RPG functions as a serial narration. Indeed, RPG proposes to explore fictional worlds by expanding narrations that can be as fertile as chaotic because, with time and practice, fictional worlds and stories can lose their coherence and versimilitude. But RPG has also some ways of regulating its expanding worlds and stories. So we are going to study how function those expanding narrations and world building and how they regulate their expansion, adopting three points of view: RPG as a media related to other media, RPG as book range and RPG as practice.

\section{INDEX}

Mots-clés : jeu de rôle, narration sérielle, expansion, régulation, monde fictionnel

Keywords : role-playing game, serial narration, expansion, regulation, world building 
AUTEUR

ISABELLE PÉRIER

3L.AM (Le Mans) et LIS (Nancy) 